\title{
Relação da grã com algumas variáveis do crescimento e propriedades da madeira de Khaya ivorensis
}

\author{
Relationship between the grain pattern and some \\ growth variables and wood properties of Khaya ivorensis
}

\author{
Graziela Baptista Vidaurre', João Gabriel Missia da Silva², Mayra de Castro3, \\ José Clailson Franco Coelho ${ }^{4}$, Alice Soares Brito ${ }^{2}$ e Jordão Cabral Moulin ${ }^{5}$
}

\section{Resumo}

Os plantios de mogno africano estão em desenvolvimento no Brasil, porém, há pouco conhecimento sobre a qualidade da madeira e sua relação com o crescimento das árvores. O objetivo deste trabalho foi avaliar a relação da grã com as variáveis dendrométricas das árvores, índices de qualidade das toras e propriedades físicas e mecânicas da madeira de Khaya ivorensis. Foram colhidas cinco árvores, aos 19 anos de idade, na Reserva Natural Vale, em Linhares, ES. A altura comercial e o diâmetro a altura do peito (DAP) das árvores foram mensurados, previamente, bem como os índices de qualidade das toras. As toras foram desdobradas em pranchões diametrais, e destes foram preparados os corpos de prova para todas as análises da madeira, em acordo com as normas vigentes. Para determinação do ângulo da grã, foram produzidos corpos de prova de $125 \mathrm{~cm}^{3}$, que foram divididos radialmente e escaneados para a mensuração do máximo desvio angular (MAD). A relação entre o MAD e as variáveis dendrométricas, índices de qualidade das toras e propriedades físico-mecânicas da madeira, foi avaliada pela correlação linear de Pearson. Observou-se a inexistência de relação entre o MAD e as variáveis dendrométricas, o índice de qualidade das toras e as propriedades mecânicas analisadas. O MAD teve uma forte relação, porém não significativa com a contração tangencial, e mediana com a densidade básica e contração longitudinal. Já a contração radial e volumétrica aumentaram proporcionalmente aos desvios da grã, situação oposta à observada para o fator anisotrópico da madeira.

Palavras-chave: Mogno africano, Máximo desvio angular, Qualidade da madeira.

\begin{abstract}
African mahogany plantations are under development in Brazil, however, there is little knowledge about wood quality and its relationship with the growth of the trees. The aim was evaluate the relationship of grain with dendrometric variables of trees, quality indexes of logs and physical and mechanical properties of wood Khaya ivorensis. Five trees were harvested at 19 years of age, in the Vale Nature Reserve in Linhares, Espírito Santo, Brazil. Commercial height and diameter at breast height (DBH) of trees were measured, previously, as well as the quality indexes of the logs. The logs were sawn in diametrical boards, and of these were prepared specimens for all the analysis of wood, according to current technical standards. To determine the grain angle, specimens of $125 \mathrm{~cm}^{3}$ were prepared, which were split radially and scanned to measure the maximum deviation angular (MAD). The relationship between MAD and the dendrometric variables, quality indexes of logs and physical and mechanical properties of wood were evaluated by Pearson correlation. We observed that there is no relationship between MAD and dendrometric variables, the quality indexes of logs and mechanical properties analyzed. The MAD had a good relationship, but not significant with the tangential shrinkage, and median with the specific gravity and longitudinal shrinkage. The radial and volumetric shrinkage increases proportion to grain deviations; an opposite situation to that observed for the anisotropic factor of wood.
\end{abstract}

Keywords: African mahogany, Maximum deviation angle, Wood quality.

Professora Adjunto no Departamento de Ciências Florestais e da Madeira. UFES - Universidade Federal do Espírito Santo / Centro de Ciências Agrárias e Engenharias. Av. Governador Lindemberg, 31 - Centro - 29550000 - Jerônimo Monteiro, ES, Brasil. E-mail: grazividaurre@gmail.com

2Doutorando(a) em Ciências Florestais. UFES - Universidade Federal do Espírito Santo / Centro de Ciências Agrárias e Engenharias. Av. Governador Lindemberg, 31 - Centro - 29550000 - Jerônimo Monteiro, ES, Brasil. E-mail: j.gabrielmissia@ hotmail.com; alice.ifnmg@yahoo.com.br

${ }^{3}$ Graduanda em Engenharia Florestal. UFES - Universidade Federal do Espírito Santo / Centro de Ciências Agrárias e Engenharias. Av. Governador Lindemberg, 31 - Centro - 29550000 - Jerônimo Monteiro, ES, Brasil. E-mail: mayra castro16@hotmail.com

${ }^{4}$ Professor substituto no Centro Multidisciplinar - Campus Floresta. UFAC - Universidade Federal do Acre. Estrada do Canela Fina, Km 12, Gleba Formoso, Lote 245 - Colônia São Francisco - 69980000 - Cruzeiro do Sul, AC, Brasil. E-mail: clailson.ufac@hotmail.com

${ }^{5}$ Doutorando em Ciência e Tecnologia da Madeira. UFLA - Universidade Federal de Lavras / Departamento de Ciências Florestais. Campus Universitário -37200000 - Lavras, MG, Brasil. E-mail: jordao_cm@hotmail.com

Sci. For., Piracicaba, v. 45, n. 114, p. 249-259, jun. 2017 DOI: dx.doi.org/10.18671/scifor.v45n114.02 


\section{INTRODUÇÃO}

A espécie Khaya ivorensis A. Chev. conhecida popularmente como mogno africano, pertence à família Meliaceae é natural da costa ocidental africana (PINHEIRO et al., 2011). A sua madeira é moderadamente estável e apresenta baixa densidade básica (0,43 a 0,49 $\left.\mathrm{g} \mathrm{cm}^{-3}\right)$ (FRANÇA et al., 2015), sendo, os principais usos: a movelaria, a produção de lâminas decorativas e instrumentos musicais, a construção naval e a arquitetura de interiores (SILVA et al., 2016). Logo, a utilização da espécie é em produtos de alto valor agregado, com uma grande exigência do mercado e da indústria por madeira padronizada e de alta qualidade (baixos índices de defeitos, propriedades físico-mecânicas estáveis e durabilidade).

Em um estudo para compreender a formação das figuras da madeira, Tulik et al. (2011) descreveram padrões únicos de cor e grã no lenho da $K$. ivorensis, que originaram um material valioso para a indústria madeireira. Os autores ressaltaram que as figuras de grã ondulada na superfície radial da madeira, derivam de alterações ondulatórias das iniciais cambiais que formam os elementos anatômicos.

No geral, a grã da madeira consiste na disposição dos elementos axiais constituintes do lenho, formada por alterações longitudinais de camadas sucessivas de células durante o crescimento da árvore (HARRIS, 1989). A madeira de K. ivorensis, frequentemente, apresenta grã irregular intercruzada, quando comparada ao mogno brasileiro (Swietenia macrophylla) (WIEMANN, 2010).

Para fins decorativos, as figuras formadas pela grã intercruzada ou ondulada são altamente valorizadas, porém podem dificultar a usinagem e ser consideradas como defeitos em madeira para uso estrutural ou com exigências de resistência e rigidez. Os desvios da grã favorecem a diminuição da resistência mecânica e a ocorrência de empenamentos e fendas, o que afeta negativamente o processamento e o acabamento da madeira serrada (FONWEBAN et al., 2013; PANSHIN; DE ZEEUW, 1980). A inclinação da grã pode reduzir a resistência à flexão e em sua direção ocorre a propagação das fendas durante a falha mecânica (KRAMER, 2006; KRETSCHMANN et al, 2010).

Na predição da resistência e rigidez da madeira de cinco espécies tropicais, por meio de métodos não destrutivos de ressonância e ultrassom, Baar et al. (2015) observaram que os desvios da grã podem reduzir a precisão na determinação do MOE dinâmico em algumas espécies.

Estudos relacionados às propriedades físicas e mecânicas da madeira de mogno africano nativa e plantada são frequentes internacionalmente. Entretanto, para a madeira proveniente de plantios em desenvolvimento no Brasil, as informações e análises sobre a sua qualidade ainda são escassas. E dentro dessa temática, não se conhece os efeitos do máximo desvio angular nas propriedades físicas e mecânicas e sua relação com as variáveis dendrométricas das árvores e índices de qualidade das toras, o que torna este trabalho pioneiro em pesquisa da grã dessa espécie plantada no país.

Neste contexto, o objetivo foi avaliar a relação do ângulo da grã com as variáveis dendrométricas das árvores, índices de qualidade das toras e propriedades físicas e mecânicas da madeira de Khaya ivorensis.

\section{MATERIAL E MÉTODOS}

Sítio e amostragem experimental: o material utilizado foi proveniente de um plantio experimental (área útil de 0,15 ha) da Reserva Natural Vale, localizada no município de Linhares, Espírito Santo, Brasil $\left(19^{\circ} 06^{\prime}\right.$ a $19^{\circ} 18^{\prime} \mathrm{S} \mathrm{e} 39^{\circ} 45^{\prime}$ a $\left.40^{\circ} 19^{\prime} \mathrm{W}\right)$. O clima local é o tropical úmido, com precipitação média anual de $1.200 \mathrm{~mm}$, temperatura média de $25,08^{\circ} \mathrm{C}$ e umidade relativa de $82,9 \%$. O espaçamento inicial do plantio foi o 3 × 2 m, sendo aplicados durante a sua implantação $200 \mathrm{~g}$ de superfosfato simples por cova.

Foram selecionadas sistematicamente, cinco árvores de K. ivorensis, com 19 anos de idade, que tiveram a altura comercial (considerada até a inserção do primeiro galho ou bifurcação) e o diâmetro à altura do peito (DAP) mensurados, previamente, com Vertex IV e suta, em ordem. Após a derrubada, as árvores foram seccionadas em toras e discos.

A primeira tora de cada árvore em virtude do diâmetro médio ser superior a $25 \mathrm{~cm}$, um padrão para serraria, foi avaliada quanto as rachaduras de topo e deslocamento de medula. Posteriormente, a primeira e segunda tora das árvores foram desdobradas para retirada de um pranchão diametral, com $8 \mathrm{~cm}$ de espessura. Nessas peças foram obtidos os corpos de prova para avaliação do máximo desvio angular (MAD) e das propriedades físicas e mecânicas da madeira (Figura 1), sendo o núme- 
ro de corpos de prova dependente das dimensões lineares dos pranchões e da ausência de defeitos (rachaduras e nós).

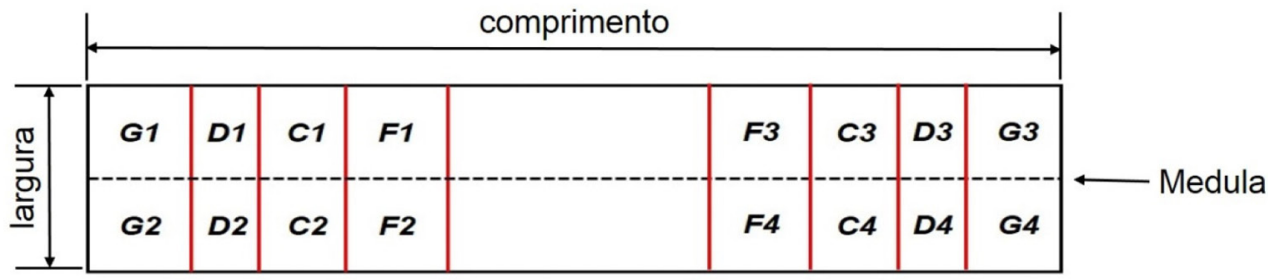

Figura 1. Esquema de amostragem do pranchão diametral para avaliação do máximo desvio angular (G); da densidade básica e contrações (D); da resistência ao cisalhamento (C) e fendilhamento (F) da madeira.

Figure 1. Sampling scheme of the diametrical board to evaluate the maximum angular deviation (G); specific gravity and shrinkage (D); shear (C) and cleavage strength (F) of the wood.

Índice de rachaduras de topo de toras (IRTT) e deslocamento da medula: essas variáveis foram utilizadas como índices de qualidade das toras. O topo das toras foi fotografado e utilizou-se o software analisador de imagens Image-Pro Plus ${ }^{\circledR}$ (versão 4.5.0.29) para a mensuração das dimensões (comprimento e abertura) das rachaduras e do deslocamento da medula, conforme o proposto por Schaitza et al. (2003) e Silva et al. (2016). De posse dessas dimensões, calculou-se o IRTT pela metodologia " $\mathrm{P}$ ", proposta por Lima et al. (2002), que considera o somatório da área das rachaduras em relação ao diâmetro da tora. O cálculo do deslocamento da medula foi feito pelo método de Lima et al. (2007).

Máximo desvio angular (MAD): foram produzidos corpos de prova nas dimensões de 50 x 50 x $50 \mathrm{~mm}$, orientados no sentido dos raios da madeira (Figura 2.A). Cada corpo de prova foi fendilhado manualmente com o uso de um facão e um martelo, de acordo com o procedimento proposto por Webb (1969) e adaptado por Hernández e Almeida (2003).
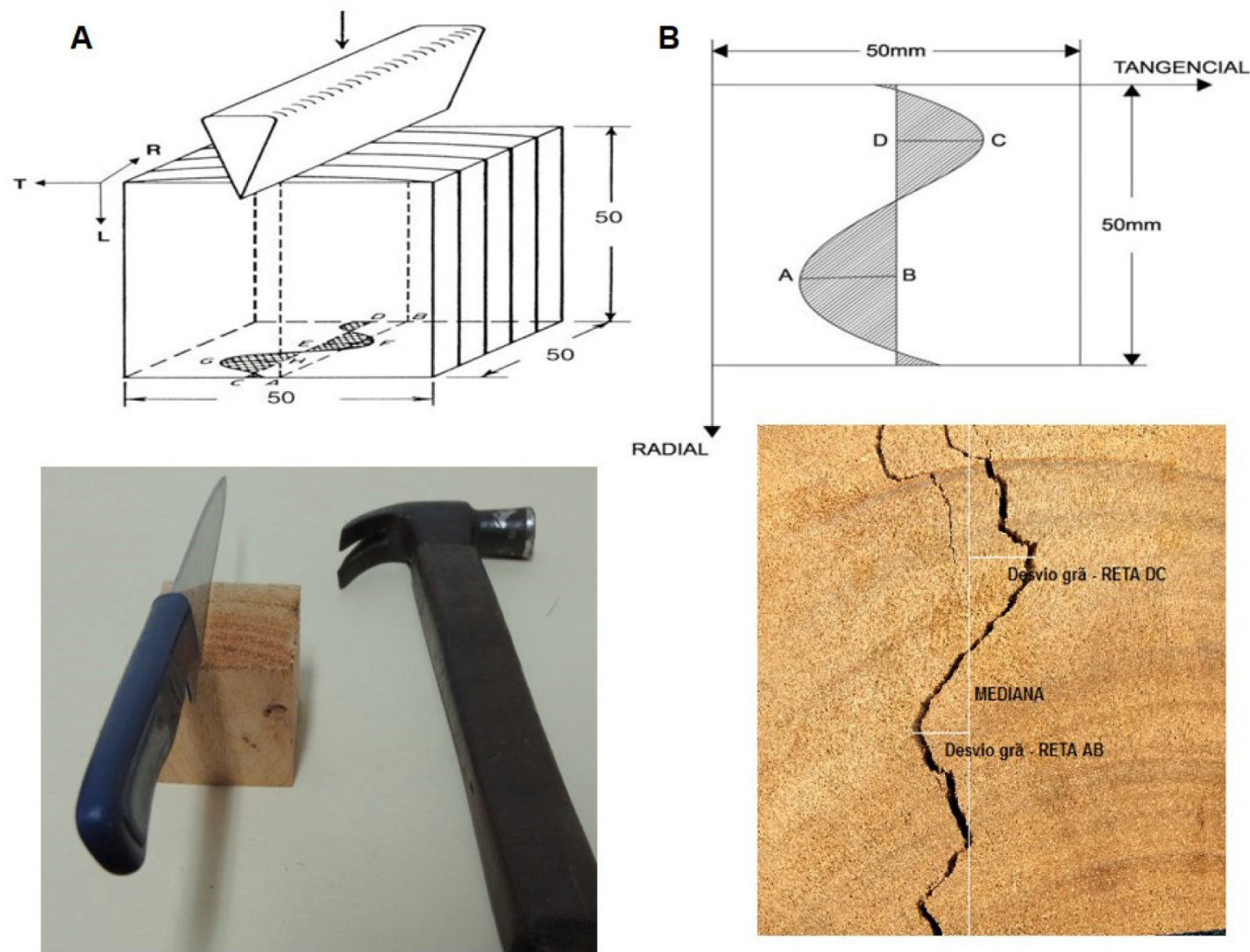

Fonte: Adaptado de Hernández e Almeida (2003).

Figura 2. (A) Direção e ferramentas para o fendilhamento dos corpos de prova; e (B) pontos de mensuração do máximo desvio angular.

Figure 2. (A) Direction and tools for cleavage of the specimens; and (B) points for measuring the maximum angular deviation. 
As amostras fendilhadas foram digitalizadas em scanner de alta resolução (600 dpi's), obtendo-se as imagens que representaram os desvios angulares formados na superfície transversal da madeira. Em seguida, realizou-se a mensuração dos desvios (Figura 2.B) com o auxílio do software analisador de imagens e pela Equação 1 obteve-se o MAD, em graus.

$$
M A D=\tan ^{-1}\left(\frac{A B}{L}\right)+\tan ^{-1}\left(\frac{C D}{L}\right)
$$

Em que: MAD: máximo desvio angular $\left({ }^{\circ}\right)$; $\mathrm{AB}$ : raio do desvio formado pela grã para o lado esquerdo $(\mathrm{cm})$; CD: raio do desvio formado pela grã para o lado direito $(\mathrm{cm})$; e L: altura da amostra $(\mathrm{cm})$.

Propriedades físicas e mecânicas da madeira: as propriedades físicas avaliadas foram: densidade básica, contrações radial, tangencial, longitudinal e volumétrica e o fator anisotrópico. Quanto às propriedades mecânicas avaliou-se a resistência ao fendilhamento e ao cisalhamento. As análises das propriedades físico-mecânicas foram realizadas, conforme os procedimentos da Norma Brasileira Regulamentadora - NBR 7190 (ABNT, 1997).

Análise estatística: todas as variáveis em estudo foram avaliadas por meio da estatística descritiva (mínimo, máximo, média e coeficiente de variação). A relação entre as variáveis dendrométricas, índices de qualidade das toras e propriedades físico-mecânicas com o MAD, foram determinadas pelo coeficiente de correlação linear de Pearson, e o seu valor P.

Dado as correlações significativas encontradas, foram ajustados modelos lineares simples $\left(Y=\beta_{0}\right.$ $+\beta_{1} \mathrm{x}+\varepsilon$ ) para estimar as propriedades de determinação mais onerosa (variável dependente) em função daquelas de análise mais simples (variável independente). Os modelos foram avaliados pela análise de variância da regressão (teste F), significância dos coeficientes estimados (teste t) e pelas medidas de precisão, coeficiente de determinação ajustado ( $\mathrm{R}^{2} a j$.) e erro padrão da estimativa (Sxy). O nível de 5\% de significância foi considerado para todos os testes.

\section{RESULTADOS E DISCUSSÃO}

A grã da madeira tem por característica ser bastante variável, tanto entre espécies, quanto na própria espécie e dentro da mesma árvore. Ao considerar o número de árvores e posições dos pranchões diametrais em que os corpos de prova foram produzidos, percebe-se que ocorreu uma alta variação entre os valores de MAD da madeira de K. ivorensis (Tabela 1). Este resultado também foi encontrado por Hernández e Almeida (2003) para as espécies Amburana cearensis, Aspidosperma macrocarpon e Clarisia racemosa, com coeficientes de variação de 30 a 39\%.

Pela ilustração dos desvios da grã na Tabela 1 é possível perceber, que com o aumento do MAD, as amostras exibiram padrões de fissuras de superfície cada vez mais irregulares, com tendência a um zigue-zague transversal, uma representação grosseira da forma de uma curva senoidal. No geral, foram produzidos vários padrões de intercruzamento nas seções transversais das amostras.

O valor médio de MAD da madeira de K. ivorensis foi próximo ao da espécie kiri (Paulownia sp.) e superior ao do cedro australiano (Toona ciliata) e da teca (Tectona grandis), que apresentaram MAD igual a $8,64,4,76$ e $4,09^{\circ}$, respectivamente (COELHO et. al., 2015). Cabrolier et al. (2009) e Hernández e Almeida (2003), encontraram valores de MAD entre 6 a $16^{\circ}$ para as espécies tropicais $A$. cearenses, A. macrocarpon, C. racemosa, Tabebuia sp., Dipterix odorata e Bagassa guianensis. Já para clones do híbrido Eucalyptus grandis x Eucalyptus urophylla aos 13 anos de idade, França (2014) observou valores de MAD variando de 6,69 a 29,80 . De tal modo, o mogno africano apresentou um valor médio de MAD próximo ao limite inferior da amplitude observada para essas espécies comerciais.

Um sistema para classificação da intensidade de intercruzamento da grã foi proposto por Limaye (1954), que considerou para a descrição de cada classe (grã direita, com tendência ao intercruzamento, moderadamente intercruzada, intercruzada, entre outras) uma amplitude limite dos desvios médios no sentido transversal das amostras. De acordo com esse sistema a madeira de K. ivorensis, aos 19 anos, foi classificada como tendo grã moderadamente intercruzada, pois o desvio médio foi igual a 0,361 $\mathrm{cm}$, inferior ao limite máximo dessa classe que é $0,476 \mathrm{~cm}$. Essa foi uma boa característica da madeira de mogno africano, pois espécies valorizadas comercialmente, como cerejeira, peroba rosa e guariuba, foram classificadas como tendo grã elevadamente intercruzada (HERNÁNDEZ; ALMEIDA, 2003). 
Tabela 1. Estatística descritiva das variáveis dendrométricas, índices de qualidade das toras, máximo desvio angular e propriedades físico-mecânicas da madeira de Khaya ivorensis aos 19 de anos de idade.

Table 1. Descriptive statistics of dendrometric variables, quality of logs, maximum angular deviation and physical-mechanical properties of wood Khaya ivorensis to 19 years old.

\begin{tabular}{|c|c|c|c|c|c|}
\hline \multirow{2}{*}{ Atributos } & & \multicolumn{4}{|c|}{ Estatística descritiva } \\
\hline & & Mínimo & Média & Máximo & Coeficiente de variação (\%) \\
\hline \multirow{2}{*}{ Variáveis dendrométricas } & $\mathrm{HC}(\mathrm{m})$ & 13,00 & 14,94 & 16,00 & 8,33 \\
\hline & $\mathrm{DAP}(\mathrm{cm})$ & 22,60 & 25,86 & 28,10 & 7,82 \\
\hline \multirow{2}{*}{ Qualidade das toras } & IRTT (\%) & 0,11 & 0,20 & 0,46 & 82,36 \\
\hline & $\mathrm{DM}(\mathrm{cm})$ & 0,91 & 2,17 & 3,37 & 47,19 \\
\hline Grã & MAD $\left({ }^{\circ}\right)$ & 3,56 & 8,25 & 19,17 & 44,99 \\
\hline \multicolumn{2}{|c|}{ Desvio mínimo } & \multicolumn{2}{|c|}{ Desvio médio } & \multicolumn{2}{|r|}{ Desvio máximo } \\
\hline \multirow{6}{*}{ Propriedades físicas } & DB $\left(\mathrm{g} \mathrm{cm}^{-3}\right)$ & 0,37 & 0,43 & 0,54 & 10,31 \\
\hline & CL $(\%)$ & 0,02 & 0,34 & 0,70 & 56,25 \\
\hline & CR $(\%)$ & 2,02 & 3,55 & 4,87 & 20,42 \\
\hline & CT $(\%)$ & 3,23 & 5,53 & 6,49 & 12,05 \\
\hline & CV $(\%)$ & 6,65 & 9,42 & 11,62 & 13,36 \\
\hline & FA & 1,14 & 1,61 & 2,54 & 19,52 \\
\hline \multirow{2}{*}{ Propriedades mecânicas } & FE (MPa) & 0,20 & 0,38 & 0,59 & 26,70 \\
\hline & $\mathrm{CS}(\mathrm{MPa})$ & 8,44 & 12,56 & 16,81 & 15,95 \\
\hline
\end{tabular}

HC: altura comercial; DAP: diâmetro a altura do peito; IRTT: índice de rachaduras de topo de toras; DM: deslocamento de medula; MAD: máximo desvio angular; DB: densidade básica; CR, CT, CL e CV: contrações radial, tangencial, longitudinal e volumétrica, em ordem; FA: fator anisotrópico; FE: resistência ao fendilhamento; e CS: resistência ao cisalhamento.

Os resultados observados para a $K$. ivorensis, juntamente com os trabalhos de Cabrolier et al. (2009), Coelho et al. (2015), França (2014); Hernández e Almeida (2003), Webb (1969) evidenciam a variabilidade e complexidade dos desvios da grã (MAD) existentes entre diferentes espécies madeireiras. Esse fato pode ser associado as características edafoclimáticas dos locais de crescimento das árvores, padrão de distribuição e direção dos ventos, taxas de incremento, práticas de manejo, características da copa e inserção de ramos laterais, inclinação do fuste e fatores inerentes a própria espécie, ou seja, características genéticas.

Essa variabilidade também pode ser atribuída à forma de avaliação do MAD, ao considerar que os desvio angulares são medidos em um único plano, quando na verdade o fenômeno é tridimensional, ou até mesmo pela heterogeneidade da estrutura microscópica da madeira entre os corpos de prova utilizados.

Para condições de corte constantes durante a usinagem, a qualidade da superfície da madeira serrada é afetada diretamente pelos desvios da grã (AGUILERA et al., 2007). Esta proposição foi confirmada durante a etapa de preparo dos corpos de prova para avaliação do MAD no mogno africano, sendo visível a olho nu na região dos maiores desvios angulares, uma superfície rugosa e com arrancamento de fibras (Figura 3). Esse efeito reduz a qualidade e torna oneroso o processamento, por causa da necessidade de operações de correção ou até mesmo, pela rejeição da peça produzida.

Ao analisar os desvios da grã da madeira associado às variáveis dendrométricas das árvores de $K$. ivorensis, observou-se a inexistência da relação do MAD com a altura comercial e o DAP (Tabela 2). Essa ausência de correlação com a altura, apesar do coeficiente mediano, pode ter sido gerada pela forma de obtenção do MAD, já que o mesmo foi avaliado em amostras retiradas apenas da primeira 
e segunda tora, e é possível que ocorram variações dos desvios angulares ao longo do fuste. Outro fator é que o pequeno intervalo de altura comercial e DAP (Tabela 1) entre as árvores de K. ivorensis e o número de indivíduos amostrados, não permitiu avaliar a relação do desvio angular com as variáveis dendrométricas em diferentes classes.

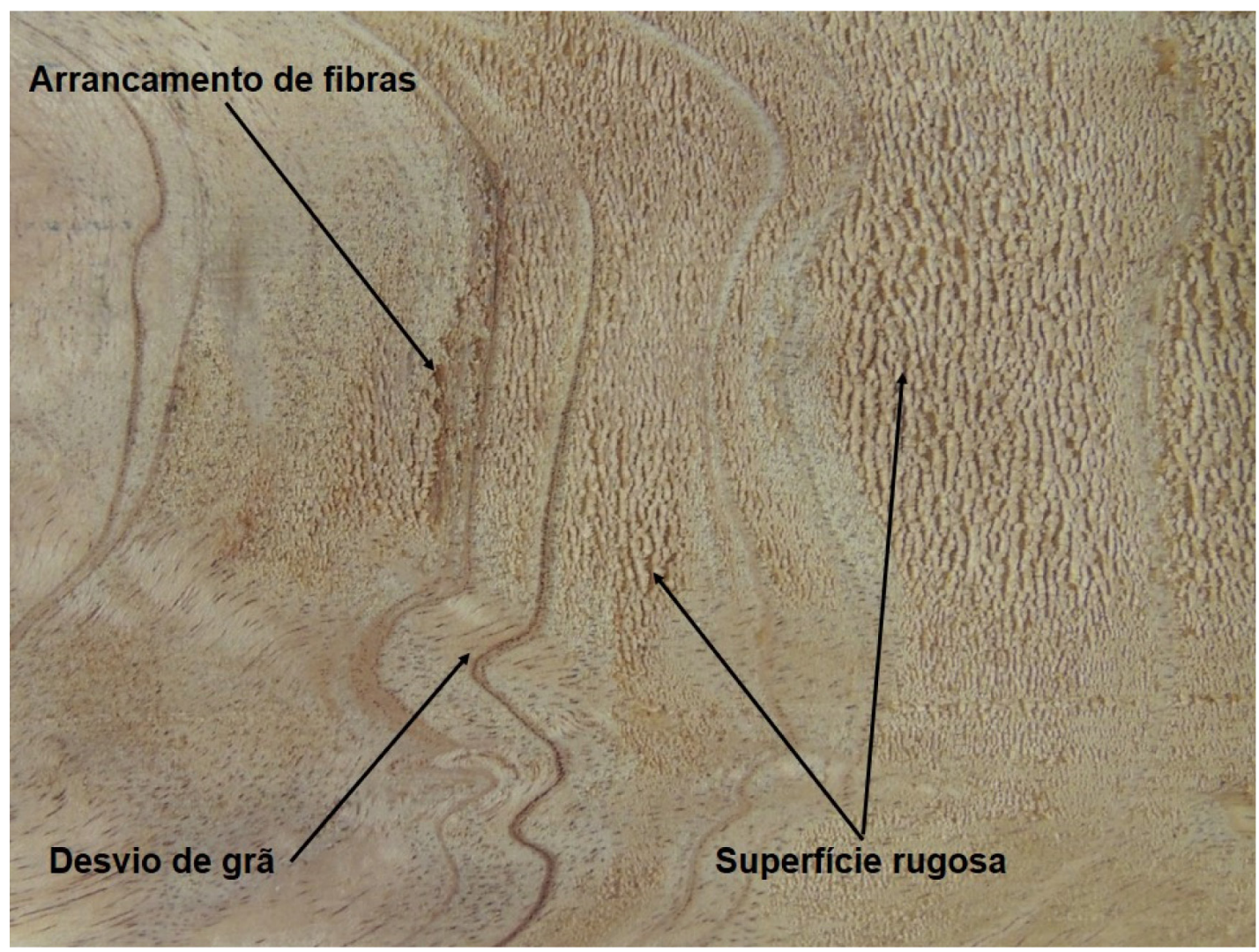

Figura 3. Efeitos dos desvios da grã na qualidade da superfície usinada da madeira de Khaya ivorensis aos 19 anos de idade.

Figure 3. Effects of grain deviations in the surface quality of machined of the wood Khaya ivorensis to 19 years old.

Tabela 2. Coeficientes de correlação de Pearson entre o máximo desvio angular (MAD) e as variáveis dendrométricas das árvores, qualidade das toras e as propriedades físicas e mecânicas da madeira de Khaya ivorensis, aos 19 anos de idade.

Table 2. Pearson correlation coefficients between the maximum angular deviation (MAD) and dendrometric variables of trees, quality of logs and the physical and mechanical properties of wood Khaya ivorensis, at 19 years old.

\begin{tabular}{lcc}
\hline Descrição das relações & Variáveis & Correlação (valor P) \\
\hline \multirow{2}{*}{ Desvios da grã associado as variáveis dendrométricas } & MAD x HC & $0,605(0,280)$ \\
\hline \multirow{2}{*}{ Desvios da grã associado a qualidade das toras } & MAD x DAP & $0,194(0,754)$ \\
\hline & MAD x IRTT & $-0,180(0,820)$ \\
& MAD x DM & $-0,905(0,09)$ \\
\multirow{2}{*}{ Desvios da grã associado as propriedades físicas da madeira } & MAD x DB & $0,610(0,275)$ \\
& MAD x CR & $0,944(0,02)$ \\
& MAD x CT & $0,822(0,09)$ \\
\hline \multirow{2}{*}{ Desvios da grã associado as propriedades mecânicas da madeira } & MAD x CL & $-0,535(0,352)$ \\
& MAD x FA & $0,916(0,03)$ \\
\hline
\end{tabular}

MAD: máximo desvio angular $\left({ }^{\circ}\right)$; HC: altura comercial (m); DAP: diâmetro a altura do peito (cm); IRTT: índice de rachaduras de topo de toras (\%); DM: deslocamento de medula ( $\mathrm{cm})$; DB: densidade básica $\left(\mathrm{g} \mathrm{cm}^{3}\right)$; $\mathrm{CR}, \mathrm{CT}, \mathrm{CA}$ e CV: contrações radial, tangencial, longitudinal e volumétrica (\%), em ordem; FA: fator anisotrópico; FE: resistência ao fendilhamento (MPA); CS: resistência ao cisalhamento (MPA). Valor P $>0,05$ não existe relação significativa entre as variáveis.

Um diagnóstico da relação do MAD com o DAP e a altura comercial é importante para prever possíveis problemas durante o processamento da madeira, por exemplo, a produção de peças serradas bem dimensionadas e em maiores bitolas, mas com acentuados desvios de grã; e para conhecer 
os reflexos da produtividade florestal nessa variável. As correlações do MAD com as variáveis de crescimento das árvores de mogno africano foram positivas, com destaque para a altura comercial, porém, elas não foram conclusivas dada a ausência de significância dos coeficientes.

Há um conflito nas relações da grã com a altura das árvores, sendo os resultados inconclusivos para diferentes espécies. Por exemplo, tendências de decréscimo e acréscimo do ângulo da grã com a altura das árvores, foram verificados por Fonweban et al. (2013) para a espécie Picea sitchensis, proveniente do norte da Grã-Bretanha. Já Krawczyszyn e Romberger (1980) apontam que o índice de grã intercruzada diminuiu com o aumento da altura do fuste de Nyssa sylvatica (35 a 80 anos). Em um clone de do híbrido E. grandis x E. urophylla aos sete anos de idade, Coelho (2016) observou uma relação diretamente proporcional em que a altura comercial das árvores explicou $76 \%$ da variação total do MAD.

O MAD não correlacionou significativamente com as rachaduras de topo das toras de K. ivorensis. Um fator que pode ter influenciado nesse resultado, é a forma de obtenção do índice de rachaduras de topo, já que o mesmo foi avaliado apenas nas extremidades da primeira tora, e as rachaduras juntamente com o MAD podem ter um comportamento diferente em maiores alturas do fuste.

Situação contrária foi observada por França (2014), que verificou correlações negativas e significativas do MAD com índice de rachaduras de topo de toras após o corte $(\mathrm{r}=-0,579 ; \mathrm{p}<0,01), 24$ horas e sete dias após o corte ( $\mathrm{r}=-0,599 ; \mathrm{p}<0,01$ e $\mathrm{r}=-0,715 ; \mathrm{p}<0,01$, respectivamente) em clones do híbrido E. grandis $x$ E. urophylla, aos 13 anos de idade. Segundo o autor, o alívio das tensões existentes nas toras ao longo do período de avaliação (logo após o corte e até sete dias após), fez com que aumentasse a correlação existente entre o MAD e o índice de rachaduras de topo, o mesmo pode ser descrito para a relação com as rachaduras nas tábuas.

Apesar de não significativa, o MAD teve uma correlação forte e negativa com o deslocamento da medula, evidenciando que as toras com medula mais excêntrica tendem a ter menores desvios da grã. Com o deslocamento da medula, ocorre um novo arranjo dos elementos celulares axiais, ficando mais paralelos ou menos inclinados em relação ao eixo longitudinal da tora, agora deslocado em relação ao centro geométrico.

Kramer (2006) afirmou que essa reorientação das iniciais fusiformes, células do câmbio, ocorre em resposta a diferentes estímulos externos e internos, gerando alterações no padrão de grã da madeira. Estudos mais detalhados dos desvios da grã em troncos inclinados ou excêntricos de mogno africano ainda são necessários.

A grã da madeira de mogno africano aos 19 anos teve uma forte relação, porém, não significativa com a contração tangencial, e mediana com a densidade básica e a contração longitudinal. Essa ausência de significância entre as propriedades mencionadas pode ser uma reposta do número de amostras utilizado, com destaque para o MAD e a contração tangencial.

A relação do MAD com a densidade básica da madeira, possuiu uma tendência de ser diretamente proporcional. Geralmente, com o aumento da espessura da parede e comprimento das fibras do lenho, ocorre um incremento na densidade. Dessa maneira, o incremento das dimensões das fibras pode ocasionar uma maior inclinação desses elementos em relação ao eixo da árvore, o que explicaria a relação de maiores desvios da grã em regiões de maior densidade da madeira. Essa relação também foi percebida em clones de eucalipto, proporcionando uma eficiente variável resposta na avaliação da qualidade da madeira dos materiais genéticos (COELHO, 2016).

Já com as contrações radial e volumétrica, a correlação foi significativa, forte e positiva, logo, com o aumento dos desvios da grã ocorreu um aumento na instabilidade volumétrica e dimensional no sentido radial. Deste modo, destaca-se a relação da anisotropia, característica da madeira, com o modelo de grã moderadamente intercruzada, peculiar ao mogno africano.

De forma geral, podemos afirmar que quanto maior o desvio da grã, maior a contração radial e volumétrica da madeira de K. ivorensis, podendo ocasionar empenamentos, torções e dificultar a trabalhabilidade desta madeira e o seu uso final. Correlações positivas entre o ângulo da grã e as contrações lineares e volumétrica $(\mathrm{r}=0,871$ a $0,936, \mathrm{p}<0,01)$ da madeira de Azadirachta indica (15 anos), foram encontradas por Sotannde et al. (2010). Para clones do híbrido E. grandis $x$ E. urophylla (13 anos) a correlação foi negativa $(\mathrm{r}=-0,584, \mathrm{p}<0,01)$ entre o MAD e a contração radial da madeira (FRANÇA, 2014). 
Diante da correlação significativa e positiva dos desvios da grã com a instabilidade dimensional da madeira, pode-se presumir que a ocorrência em ângulos extremos poderá causar parcial ou total inutilização da madeira para produtos sólidos (ZOBEL; JETT, 1995). Mediante a esse diagnóstico, torna-se cada vez mais necessária, a busca ou seleção de genótipos dessa espécie com menores desvios angulares e consequentemente maior estabilidade dimensional, o que associada a produtividade, contribuirá para um maior retorno financeiro dos plantios de mogno africano, pela oferta futura de uma madeira de melhor qualidade e que atenderá a expectativa dos consumidores.

O MAD correlacionou negativamente com o fator anisotrópico, ou seja, quanto maior o desvio da grã menor o fator anisotrópico. Esse resultado pode ser uma resposta dos valores de fator anisotrópico serem inversamente proporcionais aos de contrações radial e tangencial, tendo essas duas últimas variáveis, correlações positivas com o MAD.

Os desvios da grã da madeira de mogno africano não apresentaram correlações significativas com a resistência ao cisalhamento e ao fendilhamento, o que pode ter sido dependente do número de amostras utilizadas. Houve uma tendência, apesar de não significativa, das propriedades mecânicas serem diretamente proporcionais ao MAD, ou seja, dos desvios da grã contribuírem para o aumento da resistência ao cisalhamento e ao fendilhamento.

Nesta conjuntura, Limaye (1954) afirmou que um corpo de prova com grã intercruzada, oferece uma grande resistência à separação das fibras, ou fendilhamento, e quando ela ocorre, uma linha ondulada é formada na superfície transversal da amostra.

Quanto a relação do cisalhamento com a grã, há grandes discrepâncias entre os trabalhos da literatura (GUPTA; SINHA, 2012). Hernández e Almeida (2003) discorrem que o efeito da grã intercruzada na resistência nominal ao cisalhamento vai depender da espécie de madeira, para algumas ele é positivo e para outras negativo. Entretanto, o aumento da resistência ao cisalhamento na madeira com maiores desvios de grã, pode ser justificado pela forma em que elementos celulares estão organizados dentro do fuste ou peça serrada, já que, os mesmos se orientam em diferentes sentidos o que dificulta a formação de áreas propensas ao cisalhamento (COELHO, 2016).

Uma outra hipótese é que a inclinação da grã reduz as propriedades mecânicas da madeira, por causa do eixo das fibras não ser paralelo ao eixo geométrico das peças (GUPTA; SINHA, 2012; KRETSCHMANN et al, 2010).

As relações do MAD com outras propriedades mecânicas foram avaliadas por França (2014), Onchieku et al. (2013), Thinley et al. (2005), que observaram que o MOR e MOE foram negativamente afetados pelos desvios da grã. A mesma relação foi observada por Hernández e Almeida (2003), no estudo do efeito da grã intercruzada na resistência ao cisalhamento da madeira de A. cearensis, A. macrocarpon e C. racemosa. Uma redução de 20 a 25\% no MOE e 25 a 30\% no MOR da madeira de Fraxinus americana, Acer saccharinum e Betula alleghaniensis, foi observada para um ângulo de inclinação da grã de $10^{\circ}$ (KRETSCHMANN et al, 2010).

As formas de avaliação dos efeitos da grã nas propriedades mecânicas também causam estas diferenças. Por exemplo, alguns autores utilizam as amostras com um ângulo da grã pré-determinado na usinagem, situação diferente deste trabalho, que avaliou a relação da resistência ao cisalhamento e fendilhamento com a inclinação natural da grã nas peças de mogno africano.

Uma vez, que houve relações significativas da contração radial e volumétrica e do fator anisotrópico com o MAD, foi possível ajustar equações que estimassem essas propriedades físicas em função dos desvios da grã (Tabela 3). Ou seja, o uso dessas equações lineares permitiu estimar propriedades de determinação mais onerosa, como as contrações e o fator anisotrópico (amostragem e orientação criteriosa; demanda equipamentos para usinagem das amostras; necessidade de saturar os corpos de prova, por um período mínimo de um mês; uso de bomba de vácuo, balança, estufa e micrômetro; e maior probabilidade de erros de mensuração), em função de uma variável de análise mais simples e rápida, que é o MAD (demanda equipamentos para corte e retirada criteriosa das amostras; não há necessidade de saturação ou secagem; equipamento para obtenção das imagens, como scanner ou câmera fotográfica e um software analisador de imagens; e permite a correção dos erros de mensuração).

Todas as três equações ajustadas foram significativas e tiveram erro padrão aceitável, com coeficientes de determinação ajustado variando de 73,5 a 85,4\%. Todavia, o MAD foi mais preciso ao estimar a variação da contração radial da madeira. Os outros $14,5 \%$ da variação dessa propriedade pode ser explicado por alterações na morfologia das fibras, ângulo microfibrilar e densidade básica da madeira de K. ivorensis. 
Tabela 3. Equações ajustadas para estimar a contração radial e volumétrica e o fator anisotrópico em função do MAD da madeira de Khaya ivorensis aos 19 anos de idade.

Table 3. Adjusted equations to estimate the radial and volumetric shrinkage and the anisotropic factor according with MAD of the wood Khaya ivorensis, at 19 years old.

\begin{tabular}{lccc}
\hline Equação & $\mathbf{R}^{2}$ aj. & Sxy & P \\
\hline$C R=1,857+(0,205 \times$ MAD $)$ & 0,854 & 0,168 & $<0,05^{1}$ \\
$C V=6,506+(0,352 \times$ MAD $)$ & 0,786 & 0,361 & $<0,05$ \\
FA $=2,129-(0,0641 \times$ MAD) & 0,735 & 0,075 & $<0,05$ \\
\hline
\end{tabular}

CR e CV: contrações radial e volumétrica (\%), em ordem; MAD: máximo desvio angular ( ${ }^{\circ}$ ); FA: fator anisotrópico. R $^{2}$ aj.: coeficiente de determinação ajustado; Sxy: erro padrão da estimativa; P: valor P. ' Significativo ao nível de 5\% de probabilidade, para o teste de $F$ na análise de regressão e teste $t$ para os coeficientes da equação.

Ao considerar que a grã da madeira é controlada principalmente por fatores hereditários (HARRIS, 1988; FONWEBAN et al., 2013), a incorporação do MAD como variável para programas de melhoramento genético do mogno africano plantado no Brasil ou em outros países é viável tecnicamente. Essa ideia é corroborada por Webb (1969), que afirmou que a grã intercruzada da madeira pode ser controlada por seleção e melhoramento genético.

Entretanto, vale ressaltar que a grã também é dependente de fatores ambientais que afetam o crescimento das árvores (FONWEBAN et al., 2013), sendo de extrema importância estudos sobre os efeitos da relação genótipo $\mathrm{x}$ ambiente no MAD da madeira de diferentes espécies comerciais.

Neste contexto, o crescimento das árvores também pode ser alterado pelo espaçamento inicial de plantio e pela desrama e desbaste, sendo importante conhecer como eles podem modificar os padrões ou reduzir o ângulo da grã da madeira (FONWEBAN et al., 2013). Visto que, as florestas de mogno africano são plantadas planejando-se desbastes ao longo da rotação, a madeira ofertada em fases juvenis poderá ter maiores desvios de grã, influenciando algumas propriedades tecnológicas, sendo necessário ajustar o processamento e a secagem e buscar uma indicação de uso mais coerente.

\section{CONCLUSÕES}

O ângulo da grã influenciou a estabilidade dimensional da madeira de K. ivorensis aos 19 anos. Uma vez que, o aumento da contração radial e volumétrica e a diminuição do fator anisotrópico, foram observados com o aumento do máximo desvio angular da madeira.

As características dendrométricas das árvores, o índice de rachaduras de topo das toras e o deslocamento da medula não tiveram relação com o máximo desvio angular da madeira de K. ivorensis. Essa mesma condição também foi percebida para a densidade básica, contrações tangencial e longitudinal e propriedades mecânicas da madeira.

\section{AGRADECIMENTOS}

À Reserva Natural Vale pela concessão do material e apoio para a coleta, a Fundação de Amparo à Pesquisa e Inovação do Espírito Santo - FAPES e a Coordenação de Aperfeiçoamento de Pessoal de Nível Superior - CAPES.

\section{REFERÊNCIAS BIBLIOGRÁFICAS}

AGUILERA, A.; VEGA, M.; MÉAUSOONE, P. Effects of grain angle on the amplitudes of acoustic emission and surface roughness in wood machining. Wood Science and Technology, New York, v. 41, p. 373-381, 2007.

ABNT - ASSOCIAÇÃO BRASILEIRA DE NORMAS TÉCNICAS. NBR 7190: Projetos de estruturas de madeira. Rio de Janeiro, 1997. 107 p.

BAAR, J.; TIPPNER, J.; RADEMACHER, P. Prediction of mechanical properties - modulus of rupture and modulus of elasticity - of five tropical species by nondestructive methods. Maderas Ciencia y tecnología, Concepción, v.17, n.2, p. 239-252, 2015.

CABROLIER, P.; BEAUCHÊNE, J; THIBAUT, B. Is interlocked grain an adaptive trait for tropical tree species in rainforest? In: PLANT BIOMECHANICS CONFERENCE. 6., 2009, Cayenne. Proceedings... Cayenne: PMC, 2009.

Sci. For., Piracicaba, v. 45, n. 114, p. 249-259, jun. 2017 DOI: dx.doi.org/10.18671/scifor.v45n114.02 
Vidaurre et al. - Relação da grã com algumas variáveis do

crescimento e propriedades da madeira de Khaya ivorensis

COELHO, J. C. F. Efeitos dos desvios da grã nas variáveis dendrométricas e propriedades físico-mecânicas em madeiras de eucalipto. 2016. 60p. Dissertação (Mestrado em Ciências Florestais) - Universidade Federal do Espírito Santo, Jerônimo Monteiro, 2016.

COELHO, J. C. F.; FIRMINO, A. V.; SILVA, J. G. M.; VIDAURRE, G. B., MEDEIROS NETO, P. N.; SORANSO, D. R. Caracterização da grã da madeira de seis espécies florestais. In: CONGRESSO BRASILEIRO DE CIÊNCIA E TECNOLOGIA DA MADEIRA, 2., 2015, Belo Horizonte. Anais... Belo Horizonte: SBCTEM, 2015.

FONWEBAN, J.; MAVROU, I.; GARDINER, B; MACDONALD, E. Modelling the effect of spacing and site exposure on spiral grain angle on Sitka spruce (Picea sitchensis (Bong.) Carr.) in Northern Britain. Forestry, Oxford, v. 86, n.3, p. 331-342, 2013.

FRANÇA, F. J. N. Propriedades da madeira de eucalipto para a produção de madeira serrada. 2014. $61 \mathrm{p}$. Dissertação (Mestrado em Ciências Florestais) - Universidade Federal do Espírito Santo, Jerônimo Monteiro, 2014.

FRANÇA, T. S. F. A.; ARANTES, M. D. C.; PAES, J. B.; VIDAURRE, G. B.; OLIVEIRA, J. T. S.; BARAÚNA, E. E. P. Características anatômicas e propriedades físico-mecânicas das madeiras de duas espécies de mogno africano. Cerne, Lavras, v. 21, n. 4, p. 633-640, 2015.

GUPTA, R.; SINHA, A. Effect of grain angle on shear strength of Douglas-fir wood. Holzforschung, Berlin, v. 66, n. 5, p. 655-658, 2012.

HARRIS, J. M. Spiral grain and wave phenomena in wood formation. Berlin / New York: Springer, 1989. $215 \mathrm{p}$.

HERNÁNDEZ, R.; ALMEIDA, G. Effects of wood density and interlocked grain on the shear strength of three Amazonian tropical hardwoods. Wood and Fiber Science, Madison, v. 35, n. 2, p. 154-166, 2003.

KRAMER, E. M. Wood Grain Pattern Formation: A Brief Review. Journal Plant Growth Regulation, v. 25, n. 4, p. 290-301, 2006.

KRAWCZYSZYN, J.; ROMBERGER, J. A. Interlocked Grain, Cambial Domains, Endogenous Rhythms, and Time Relations, with emphasis on Nyssa sylvatica. American Journal of Botany, Columbus, v. 67, n. 2, p. 228236, 1980.

KRETSCHMANN, D. E.; BRIDWELL, J. J.; NELSON, T. C. Effect of changing slope of grain on ash, maple, and yellow birch bending strength. In: WORLD CONFERENCE ON TIMBER ENGINEERING, 11., 2010, Riva del Garcia, Italia. Proceedings... Riva del Garcia, WCTE, 2010.

LIMA, I. L.; GARCIA, J. N.; PIEDADE, S. M. S. Rachaduras de extremidades de tora e suas implicações nas rachaduras da madeira serrada. Scientia Forestalis, Piracicaba, n. 61, p. 13-24, 2002.

LIMA, I. L.; GARCIA, J. N.; STAPE, J. L. Influência do desbaste e da fertilização no deslocamento da medula e rachaduras de extremidade de toras de Eucalyptus grandis Hill ex-Maiden. Cerne, Lavras, v. 13, n. 2, p. 170$177,2007$.

LIMAYE, V. D. Interlocking of grain in Indian timbers. Indian Forester, Dehradun, v. 80, n. 1, p. 6-9, 1954.

ONCHIEKU, J.; GITHIOMI, J. K.; OBALLA, P.; CHAGALLA-ODERA, E. Effect of Spiral Grain Occurrence on Strength Properties of Pinus patula Grown in Kenya. International Journal of Applied Science and Technology, v. 3, n. 2, p. 36-43, 2013.

PANSHIN, A. J.; DE ZEEUW, C. Textbook of wood technology: structure, identification, properties and uses of the commercial woods of the United States and Canada. 4. ed. New York: McGraw- Hill, 1980. 722 p. 
PINHEIRO, A. L.; COUTO, L.; PINHEIRO, D. T.; BRUNETTA, J. M. F. C. Ecologia, silvicultura e tecnologia de utilização dos mognos-africanos (Khaya spp.). Viçosa, MG: Sociedade Brasileira de Agrossilvicultura, 2011. $102 \mathrm{p}$.

SCHAITZA, E. G.; MATTOS, P. P.; PEREIRA, J. C. D. Metodologias simples e baratas para análise de Imagens planas. Colombo: EMBRAPA, 2003. 4 p. (Comunicado técnico, 90)

SILVA, J. G. M.; VIDAURRE, G. B.; ARANTES, M. D. C.; BATISTA, D. C.; SORANSO, D. R.; BILLO, D. F. Qualidade da madeira de mogno africano para a produção de serrados. Scientia Forestalis, Piracicaba, v. 44, n. 109, p. 181 - 190, 2016.

SOTANNDE, O. A.; OLUYEGE, A. O.; ADEOGUN, P.F.; MAINA, S.B. Variation in Wood Density, Grain Orientation and Anisotropic Shrinkage of Plantation Grown Azadirachta Indica. Journal of Applied Sciences Research, v. 6, n. 11, p. 1855-1861, 2010.

THINLEY, C.; PALMER, G.; VANCLAY, J. K.; HENSON, M. Spiral and interlocking grain in Eucalyptus dunii. European Journal of Wood and Wood Products. v. 63, n. 5, p. 372-379, 2005.

TULIK, M.; KLOSINSKA, T.; IQBAL, M.; GROCHOWINA, A. Figures of the Wood of Khaya Ivorensis and Millettia laurentii. Wood Research, Bratislava, v. 56, n. 4, p. 613-620, 2011.

WEBB, C. D. Variation of interlocked grain in Sweetgum. Forest Products Journal, Madison, v. 19, n. 8, 1969.

WIEMANN, M. C. Characteristics and availability of commercially important woods. In: Forest Products Laboratory - FPL. Wood Handbook: wood as an engineering material. Madison: FPL/USDA, 2010, Cap. 1, p. 16-60.

ZOBEL, B. J.; JETT, J. B. Genetics of wood production. New York: Springer series in wood science, 1995.337 p.

Recebido em 22/04/2016

Aceito para publicação em 09/01/2017

Sci. For., Piracicaba, v. 45, n. 114, p. 249-259, jun. 2017 DOI: dx.doi.org/10.18671/scifor.v45n114.02 
